\title{
An Absolute Radiometer Based on InP Photodiodes
}

\author{
Ana Luz Muñoz Zurita ${ }^{1}$, Joaquin Campos Acosta ${ }^{2}$ \\ and Alicia Pons Aglio ${ }^{2}$ \\ ${ }^{1}$ Autonomous University of Coahuila \\ Faculty of Engineering Mechanical and Electrical U Torreon \\ ${ }^{2}$ CSIC- Institute for Applied Physics \\ ${ }^{1}$ Mexico \\ ${ }^{2}$ Spain
}

\section{Introduction}

Semiconductor photodetectors based on InP materials are the ones most often used in state of the art long wavelength optical fiber communication system. Mixed compounds such as InGaAs $(\mathrm{P})$ and $\mathrm{In}(\mathrm{Al}) \mathrm{GaAs}$ lattice matched to $\mathrm{InP}$ are the materials responsible for detecting long wavelength light, specially the nondispersion wavelength $(1.3 \mu \mathrm{m})$ and loss minimum wavelength $(1.55 \mu \mathrm{m})$ of silica optical fibers. The characteristics of these InP-based photodectors are superior to those of conventional photodiodes composed of elemental Ge, which was the only material applicable for wavelengths below $1.55 \mu \mathrm{m}$. By using a heteroestructure, which hadn't been expected in group IV elemental semiconductors such as $\mathrm{Si}$ and Ge, new concepts and new designs for high performance photodetectors have been developed. For example, the absorption region can be confined to a limited layer and the InP wide bandgap layer can serve as a transparent layer for specific communication wavelength. Recently InGaAs/InP avalanche photodiodes (APDs) with a SAM (separation of absorption and multiplication) configuration have become commercially available. The SAM configuration is thought to be necessary for high performance APDs utilizing long wavelengths.

The photodiodes may be operated under reverse bias, high quality semiconductor layers need to be produced. To obtain photodiodes that operate at a low bias and have a low dark current, it is necessary to produce epitaxial layers that are pure and have few defects (such as dislocations, point defects, and impurity precipitates). To get stable and uniform gain in APDs, in which internal gain is achieved through the carrier avalanche process, the layers in the avalanche region must be uniform and free of dislocations. Furthermore, a planar device structure requires that a guard ring be used to keep the electric field around the photoreceptive area from increasing too much. Fabrication and processing technologies such as impurity diffusion, ion implantation, and passivation will also play important roles in the production of reliable photodetectors.

From a radiometric point of view, the photodetectors important characteristics are: Speed of (characterized by the bandwidth of the frequency response or the Full Width Half 
Maximum (FWHM) of the pulse response), responsivity (determined as the ratio of current out the detector to the incident optical power on the device), sensitivity (defined as the minimal input power that can still be detected which, as a first approximation, is defined as the optical power which generates an electrical signal equal to that due to noise of the diode). One related characteristic is the quantum efficiency of the detector which is the ratio of the number of electron-hole pairs which contribute to current to the number of incident photons.

When the light radiation impinges on a detector, various physical processes occur; part of the incident light is reflected by the sensitive surface, while the rest passes inside the detector, where can be partially, because of losses due to absorption, converted into an electronic signal. The response of each photodetector is conditioned by a quantity of the converted light power, but for evaluating the incident power one has to know the ratios of the reflected, absorbed, and converted portions.

The InP photodetectors are chosen as the first device of interest because of their simple structure, and since their analysis is a natural extension, almost an example, of our discussion of $\mathrm{p}-\mathrm{n}$ diodes. Whereas the field of photodetectors goes far beyond that of semiconductor photodetectors, we restrict ourselves here to such devices. It will be discussed p-i-n diodes, which are also referred to as photovoltaic detectors, photoconductors or solar cells photodetectors. The distinction between the different devices is somewhat artificial since many similarities exist between these devices but it enables to clearly separate the difference in structure, principle of operation and purpose of the devices.

InP-photodiodes from different manufacturers have got rather low noise level, a good response uniformity over the sensitive surface and a wide dynamic range. Therefore they are good devices to built radiometers in the NIR spectral region. As in any photodiode, the spectral short-circuit responsivity is determined by the wavelength and the photodiode's reflectance and internal quantum efficiency. Then if these quantities were known, the photodiode's responsivity would be known without being compared to another standard radiometer; i. e. the photodiode would be an absolute radiometer for optical radiation measurements.

This idea was firstly developed for silicon photodiodes in the eighties, once the technology was able to produce low defects photodiodes. Following this reference, the reflectance could be approached from a superimposed thin layers model. By knowing the thicknesses of the layers and the optical constants of the materials, it is possible to determine the device reflectance. However, this information is not completely available for InP photodiodes: the actual thickness of the layers is not known and optical constants of materials are only approximately known for bulk. Nevertheless it's possible to measure reflectance at some wavelengths and to fit the thicknesses of a layer model that would reproduce those experimental values.

The internal quantum efficiency cannot be determined.Since InP photodiodes are heterojunctions rather than homo-junctions as silicon photodiodes are. In the other hand, since the internal structure is not accurately known, it is not possible to model the internal quantum efficiency without having experimental values for it.

Therefore the attainable scope at present is just to obtain a model to be able to calculate spectral responsivity values at any wavelength. To get this, a model has been developed to calculate reflectance values from experimental ones at some wavelengths and another 
model has been developed to interpolate spectral internal quantum efficiency values from some values got from reflectance and responsivity measurements at some wavelengths. Both models will be presented in this chapter.

\section{Pin-photodiode design, fabrication and modelling}

Photodiodes with different lengths from $30 \mu \mathrm{m}$ to $70 \mu \mathrm{m}$ and width $8 \mu \mathrm{m}$ were fabricated to analyse their RF behavior. A schematic view of the pin photodetector is depicted in figure 1 . The structure of the pin-photodiode is based on a transverse twin-guide scheme. From the top down, the main parts of the pin-photodiode are a $400 \mathrm{~nm}$ p+ -InP contact layer, $100 \mathrm{~nm}$ InGaAs absorbtion layer, $100 \mathrm{~nm}$ non-intentionally doped and $400 \mathrm{~nm} \mathrm{n}+-\operatorname{InGaAsP}(\mathrm{Q} 1.3)$ layer, acting as the top waveguide layer and as n-contact layer. With this structure layer and $8 \times 60 \mu \mathrm{m} 2$ area $0.25 \mathrm{pF}$ can be achieved for a reverse bias voltage of $2 \mathrm{~V}$ and $0.12 \mathrm{pF}$ for $-5 \mathrm{~V}$. The access waveguides consist of a $600 \mathrm{~nm} \mathrm{Q(1.3)} \mathrm{layer} \mathrm{with} \mathrm{an} \mathrm{InP}$ cladding layer which is tapered from the normal width of $2 \mu \mathrm{m}$ to $8 \mu \mathrm{m}$.

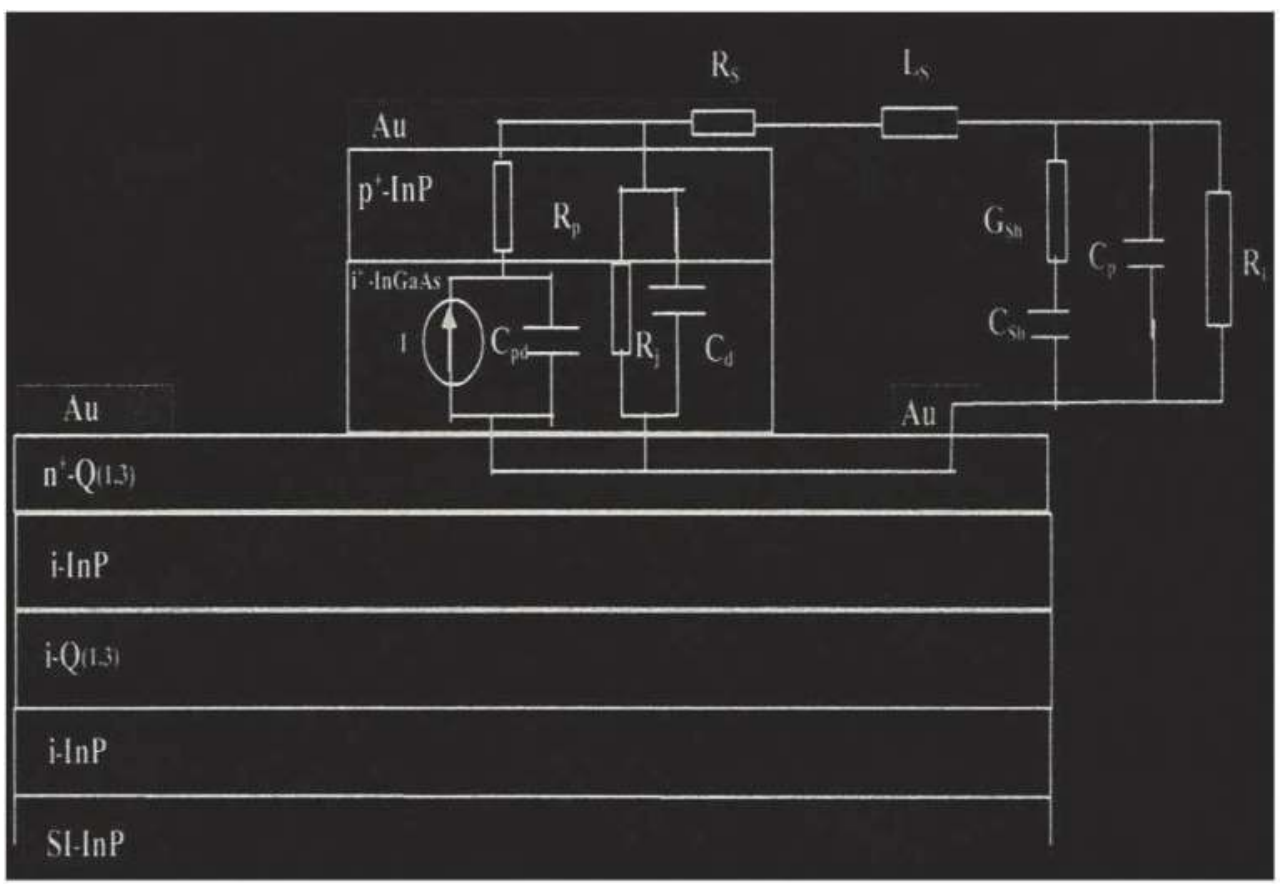

Fig. 1. Cross section of a side-illuminated twin-waveguide pin-photodiode. The equivalent circuit elements represent physical parameters of the photodiode and parasitic elements.

Electrically, the output of the pin photodiode is fabricated as a coplanar waveguide transmission line in order to facilitate hybrid integration with the GaAs travelling-wave amplifier and also for on-wafer high-frequency measurements. Figure 1 indicates how the parasitic components are connected and how they are related to the physical parameters of the pin photodiodes. 
Here Rs is the $p$ - and n-electrode contact resistance, $R \mathrm{j}$ is the resistance representing the dark current leakage, $\mathrm{R} \mathrm{p}$ is the resistance of the bulk P+ -InP layer; Cpd is the reverse-bias junction capacitance, $\mathrm{Cd}$ capacitance between $\mathrm{p}$ - and $\mathrm{n}$-contact metallization which is significant at low frequencies, Ls is the inductance of the $\mathrm{p} / \mathrm{n}$-contacts to signal/ground electrodes, Csh and Gsh are the shunt capacitance and conductance of coplanar waveguide which take into account the electric energy storage inside the transmission line and the losses caused by the transverse component, $\mathrm{Cp}$ is the open circuit at the end of the coplanar waveguide transmission line, and RL is the load resistance.

In the case of semiconductors photodetectors based on InP materials are the ones most often used in state of the art long-wavelength optical fiber communication systems. Mixed compounds such $\operatorname{InGaAs}(\mathrm{P})$ and $\operatorname{In}(\mathrm{Al}) \mathrm{GaAs}$ lattice matched to $\mathrm{InP}$ are the materials responsible for detecting long-wavelength light, especially the nondispersion wavelength $(1.3 \mu \mathrm{m})$ and loss minimum wavelength $(1.55 \mu \mathrm{m})$ of silica optical fibers.

The characteristic of these InP based photodetectors are superior to those of conventional photodiodes composed of elemental Ge, which was the only material applicable for wavelengths below $1.5 \mu \mathrm{m}$. By using a heterostructure, which had not been expected in group IV elemental semiconductor such as $\mathrm{Si}$ and $\mathrm{Ge}$, new concepts and new designs for high-performance photodetectors have been developed. For example, the absorption region can be confined to a limited layer, and the InP wide-bandgap layer can serve as a transparent layer for specific communication wavelength. Recently InGaAs/InP avalanche photodiodes (ADPs) with a SAM (separation of absorption and multiplication) configuration have become commercially available. The SAM configuration is thought to be necessary for high performance APD’s utilizing long wavelenghts.

Because photodiodes operate under reverse-bias, high quality semiconductor layers need to be produced. To obtain photodiodes that operate at a low bias and have a dark current, it is necessary to produce epitaxial layers that are pure and that have few effects (such as dislocations, points defects and impurity precipitates).To get stable and uniform gain in APDs, in which internal gain is achieved through the carrier avalanche process, the layers in the avalanche region must be uniform and free of dislocations. Futthermore a planar device structure requieres that a guardring be used to keep the electric field around the photoreceptive area from increasing to much.

Fabrication and processing technologies such as impurity diffusion, ion implantation and passivation will also play important role in the production of reliable photodetectors. The photodiodes used in received circuits are required to traslate optical signals into electrical signals faitfully and efficiently.

The reflectance and the internal quantum efficiency are determined the photodiode spectral responsivity, which is the radiometric characteristic of interest in the fields where these devices can be used for optical radiation measurements. It presents the experimental set up for measuring the photodiode reflectance as well as the results of such measurements related to InGaAs/InP-photodiodes exploited in international laboratories.

The obtained experimental results show that some models of photodiodes have got an antireflecting coating on their sensitive facets and that reflectance does not change with varying the light polarization state within the measurement uncertain, when the angles of incidence are less that $7.4^{\circ}$.

\section{Applications of InP photodiodes}

Future systems based on microwave and optics such as fiber optic radio communications will require very high speed InP photodetectors able to work in the microwave or millimeter 
wave frequency range. It is well-known that PIN photodetectors limitations are due to transit time and capacitance.

To increase their cut-off frequency, it is necessary to reduce transit time, so thickness of absorbing layer, and capacitance. For top-illuminated PIN photodetectors, this will decrease dramatically the responsivity of the photodetector, and theses detectors are generally used up to around $20 \mathrm{GHz}$, as maximum bandwidth. For upper frequencies, the PIN waveguide photodetector is an attractive device, since it is possible to reduce transit time without decreasing responsivity, because of the absorbing core waveguide structure. Moreover, the optical signal is absorbed over a short length $(5-10 \mu \mathrm{m})$, and the device , so the capacitance, can be very small. Recently, demonstrations with high responsivity up to $60 \mathrm{GHz}$ at Thomson LCR1 and even at higher millimeter wave frequencies2 were performed with device grown on semi-insulating InP substrate. Microwave access is a coplanar line to reduce parasitics, and the waveguide is a multimode structure to improve the optical coupling with the optical fiber. Interdigitated Metal Semiconductor Metal (MSM) photodetectors on AlInAs/GaInAs/InP epilayers were also subject to attention because of their low capacitance.

Commercially available MSM (New Focus) were developed with $40 \mathrm{GHz}$ cut-off frequency. Nevertheless, as for PIN photodetectors, high speed MSM photodetectors require thin absorbing layers together with short electrode spacing, so reduced responsivity. Solution would be waveguide devices, or MSM coupled with optical waveguide, but the technology is then more complicated, and the PIN waveguide photodetector remains the best solution.

PIN photodiode (or MSM photodetector) does not exhibit internal gain. It is the reason why heterojunction phototransistor (HPT) in the InP/InGaAs material system has been studied for several years. HPTs could be a good alternative to top illuminated or edgecoupled PIN photodiodes since they exhibit an internal gain owing to transistor effect, without high bias voltage as required for avalanche photodiodes and without excess noise due to avalanching. If we do not consider doping of epilayers, epitaxial structure of HPT is very similar to the one of PIN waveguide photodiode. It means that waveguide technique could also be interesting to apply to HPTs for microwave or millimeter wave applications.

Photodiodes are semiconductor devices responsive to high energy particles and photons. Photodiodes operate by absorption of photons or charged particles and generate a flow of current in an external circuit, proportional to the incident power. Planar diffused silicon photodiodes are P-N junction diodes. A P-N junction can be formed by diffusing either a Ptype impurity, such as Boron, into a N-type bulk or epitaxial silicon wafer, or a N-type impurity, such as Phosphorus, into a P-type bulk or epitaxial wafer. The diffused area defines the photodiode active area. To form an ohmic contact, another impurity diffusion into the backside of the wafer is necessary. The active area is coated with an Anti-Reflection coating to reduce the reflection of the light for a specific predefined wavelength. The $\mathrm{P}$ and $\mathrm{N}$-sides of the junction have metal pads, which make an electrical contact through dielectric layers.

Due to the high absorption coefficient, the InGaAs absorption region is typically a few micrometers thick. The thin absorption layer enables the device to obtain high speed at a low reverse bias voltage, typically 2-5 volts. The InP window layer is transparent to $1.3 \mu \mathrm{m}-$ $1.55 \mu \mathrm{m}$ wavelengths, thus InGaAs/InP photodiodes do not have slow tail impulse response associated with the slow diffusion component from the contact layer. 


\section{Basic photodiode operation}

Photodiodes operate under reverse vias to create a depleted region in which photogenerated electron-hole pairs. In the figure 2 show a schematic cross section of a photodiode ( or avalanche) with a $\mathrm{p}(+),(-) \mathrm{n}, \mathrm{n}(+)$ structure. It also show the optical absorption, which is subject to the absorption coefficient of the material for incident light and decreases exponentially with increasing distance from the diode front $\mathrm{p}(+)$.

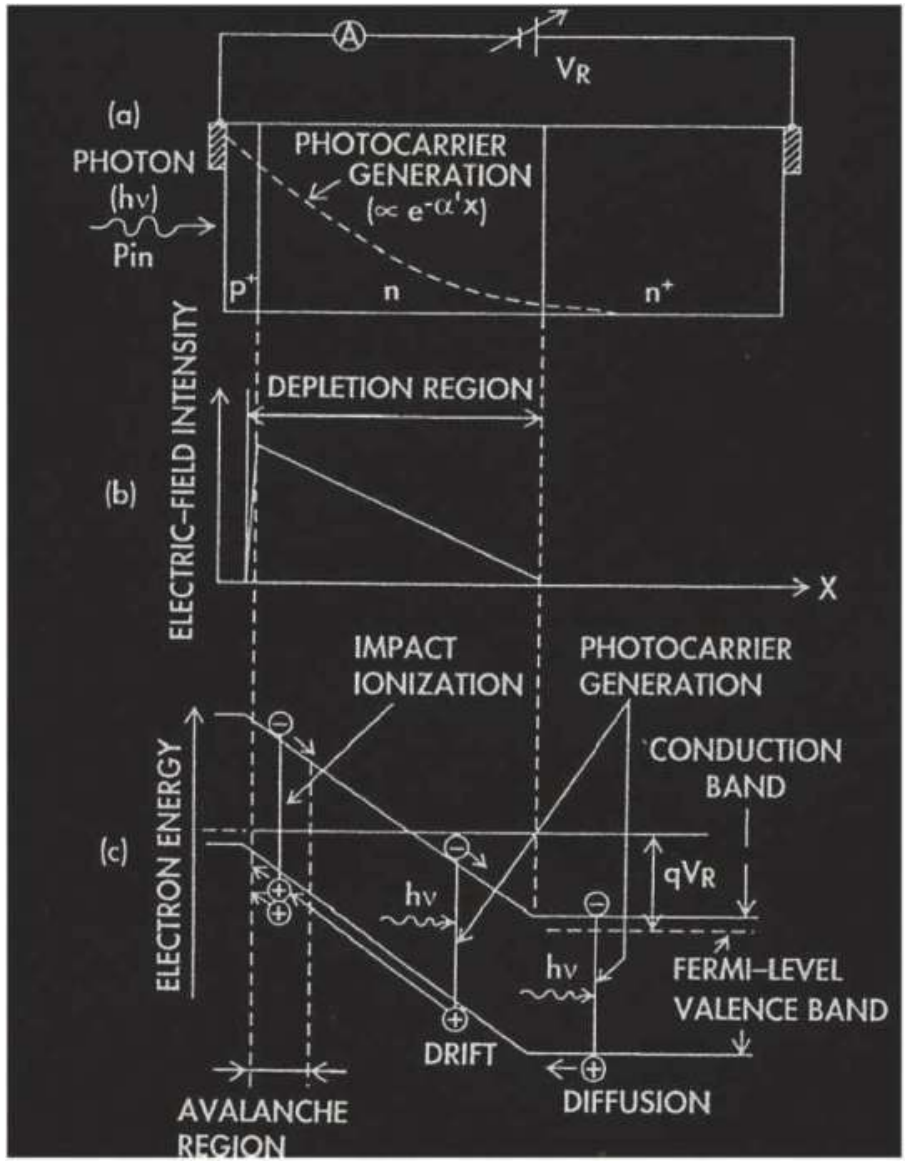

(a) Cross sectional sketch of $\mathrm{p}(+), \mathrm{n}(-), \mathrm{n}(+)$ diode under reverse bias.

(b) Electric field distribution

(c) Energy band diagram

Fig. 2. Basic operation of a Photodiode.

In the Figure 2(b) and (c) show electric field distribution and the energy band diagram, respectively. Most photocarriers are designed for use in the fully depleted n-region, so that they have a high-speed response: Electrons and holes generated within the depletion region are instantaneously separated by the elelectrical field and drift in the opposite direction, 
inducing a photocurrent in the external circuit. On the other hand, minority-carrier holes excited within an average diffusion length in the undepleted $n(+)$ region adjacent to the depleted region diffuse into the edge of the depleted junction with some recombination and are colleted across the high-field region, resulting in a diffusion photocurrent in the external circuit. Diffusion photocurrent is generally characterized by its slow response to the optical signal, since the speed of the response depends on the time it takes for the photogenerated minority carriers to diffuse from where they are generated in the neutral undepleted region into the edge of the depletion region.

The photodiode should therefore be designed in such a way that there is no optical absorption in the undepleted neutral region. For the same reason, as well as to reduce the recombination loss of photocarriers genereted in the $\mathrm{P}(+)$ region on the fron side of the diode, the $\mathrm{p}(+)$ region must be as thin as possible.

When the electric field of photodiode is elevated to several hundreds of kilocolts per centimeter by increasing reverse bias, as internal gain for a primary photocurrent can be obtained. This gain is a result of the electron-hole pair creation is denominated avalanche process, initiated by the photogenerated carriers, which creation is in turn governed by the relation between the strenght of the electric field and the electron and hole impact ionization rates of the material itself.

\subsection{Basic InP PIN photodiodes}

In making a simple planar structure the photodiode a double heterostructure consisting of InGaAs/InP with a inP capping layer is grown, and this growth is followed by selective impurity diffusion to form the $\mathrm{p}(+) \mathrm{n}(-)$ junction. A window allowing light to pass through is formed on the front ( the grown-layer superffice) or back surface of the InP substrate. The back-illumination type is of a structure often used to obtain the low capacitance desirable for high-speed operation.

A cross -sectional view of a front-illuminated planar structure InGaAs/InP - PIN photodiode is show in the figure 3.

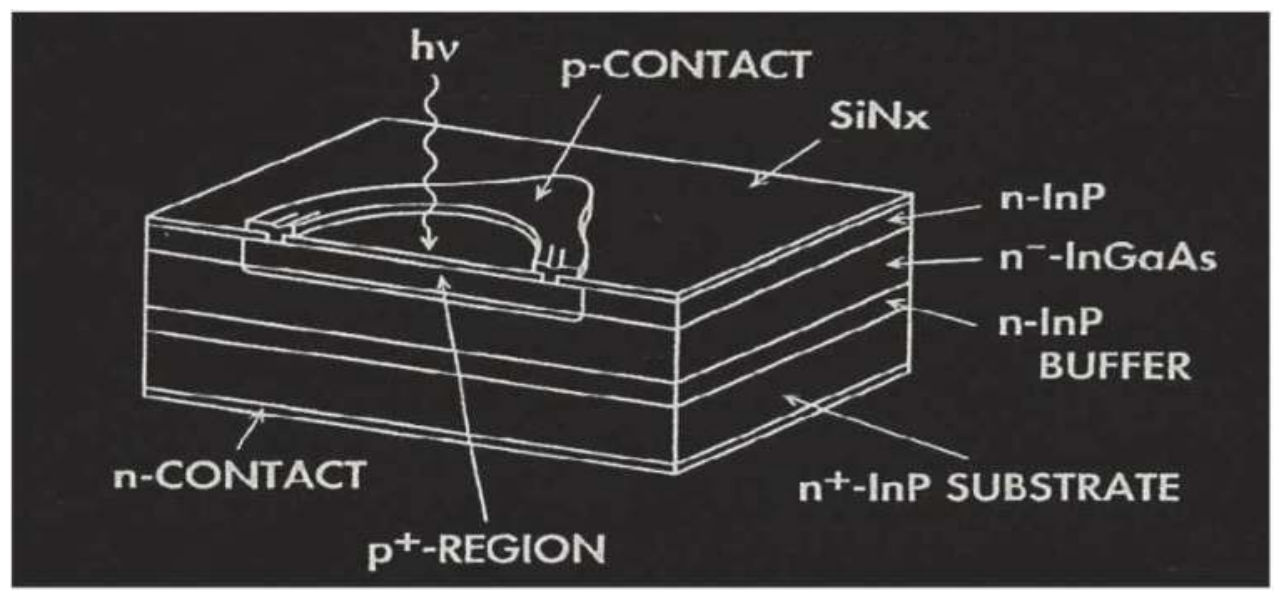

Fig. 3. Schematic cross section of a planar structure InP Photodiode. 
In the case of InP Photodiodes we need to check something important parameters for example:

a. Receiver Sensitivity.

b. Response Speed.

c. Dark Current Reduction.

d. High Speed and high quantum efficiency.

e. Reduction of diode capacitance.

f. Large-ionization rate ratio materials for the avalanche layer.

\section{InP-photodetectors}

At the present time, the InGaAs/InP-photodetectors from different manufactures have rather low level of noise, a good uniformity of the surface response as well as a wide dynamic range and linearity.

For these reasons they are exploited in the instruments for measuring optical radiation within the near infrared (IR) range $(800-1600 \mathrm{~nm})$. Furthermore, the InGaAs/InPphotodetectors are used for maintaining the scale of spectral responsitivity in the same spectral range in many laboratories. It is presented this chapter devoted to studying the reflectance of photodiodes from different manufactures.

For this reason, measuring the reflectance of photodiodes is presented in this chapter as a preliminary step to finding the responsivity. The photodiode response depends on a set of parameters inherent in the incident light like the spectral distribution, polarization, modulation of frequency, angle of incidence, and radiant power.

Then, the response is determined by such characteristics of photodetector as the material refraction index and the structure of diode as well as by some environmental factors, for example, by the temperature.Photodiodes are semiconductor devices responsive to high energy particles and photons. Photodiodes operate by absorption of photons or charged particles and generate a flow of current in an external circuit, proportional to the incident power. Planar diffused silicon photodiodes are P-N junction diodes. A P-N junction can be formed by diffusing either a P-type impurity, such as Boron, into a N-type bulk or epitaxial silicon wafer, or a N-type impurity, such as Phosphorus, into a P-type bulk or epitaxial wafer. The diffused area defines the photodiode active area. To form an ohmic contact, another impurity diffusion into the backside of the wafer is necessary. The active area is coated with an Anti-Reflection coating to reduce the reflection of the light for a specific predefined wavelength. The $\mathrm{P}$ and $\mathrm{N}$-sides of the junction have metal pads, which make an electrical contact through dielectric layers.

Due to the high absorption coefficient, the InGaAs absorption region is typically a few micrometers thick. The thin absorption layer enables the device to obtain high speed at a low reverse bias voltage, typically $2-5$ volts. The InP window layer is transparent to $1.3 \mu \mathrm{m}-$ $1.55 \mu \mathrm{m}$ wavelengths, thus InGaAs/InP photodiodes do not have slow tail impulse response associated with the slow diffusion component from the contact layer.

\section{Experimental procedure}

To realize our experiments related to measuring the reflectance of InGaAs/InP photodiodes it have arranged the experimental set-up presented in Figure 4. 


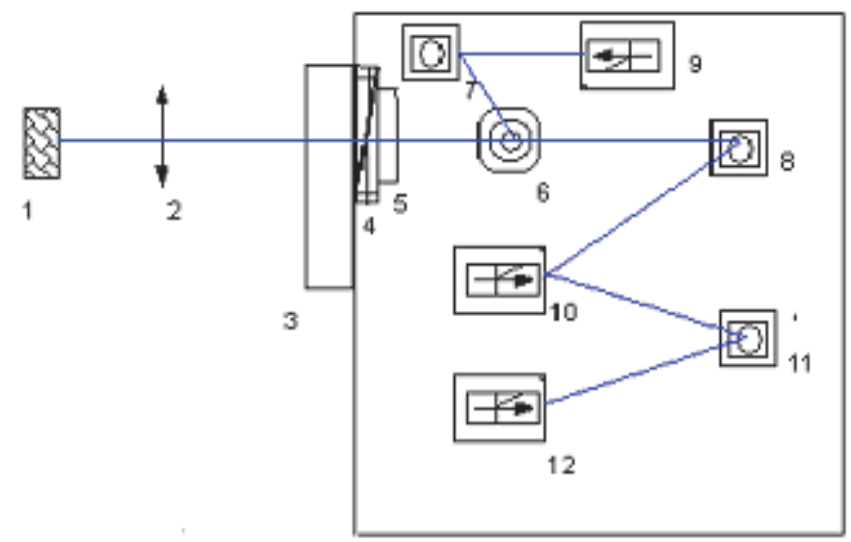

1. Source light (Incadescent lamp)

2. Lens

3. Monochromator

4. Polarizer

5.Stopper (Choper)
6. Beam splitter AR34

7,8,11. Mirrors

9,12 . Germanium detectors

10. $\mathrm{InGaAs} / \mathrm{InP}$ detectors with is exchanged with standart mirror BK47

Fig. 4. Experimental set-up for measuring the reflectance InGaAs/InP photodiodes

It has exploited an incandescence lamp is the source of white light imaged at the input slit of the monochromator. This lamp was able to cover the spectral range from 800 to $1600 \mathrm{~nm}$ and had appropriate blocking filters for second - order wavelengths. After the monochromator, it had placed a linear polarizer and a beam splitter, which serves to monitor temporal fluctuations of light power. A germanium photodiode was used as the monitoring reference photodetector.

The experimental set-up included an optical system of mirrors, which consists of two parts. An upper part (see mirror 7 and germanium photodiode 9) realized monitoring temporal fluctuations of light power. A bottom part (see mirrors 8, 11; InGaAs/InP-photodiode 10, and and germanium photodiode 12) formed an image of the monochromator's exit slit on 
the sensitive surfaces of photodiodes. The angle of incidence was equal to 7.4 grades which was accepted as the normal incidence in this train of measurements. The method of measurement consists in comparing the response from a germanium photodiode to the radiation reflected by the $\mathrm{InGaAs} / \mathrm{InP}$ photodiode with the response from an aluminium standard mirror whose reflectance is known, so that :

$$
P(\lambda) \equiv \frac{I_{p}(\lambda)}{I_{m}(\lambda)} \rho_{m}(\lambda)
$$

Here, $I_{p}(\lambda)$ is the response to the light reflected by the $\operatorname{InGaAs} / \operatorname{InP}, I_{m}(\lambda)$ is the response to the light reflected by the mirror, and $\rho_{m}(\lambda)$ is the reflectance of a standard mirror. With this method it has measured the reflectance of photodiodes from different manufacturers. One part of detectors had a round aperture of $5 \mathrm{~mm}$ in diameter and the other part had a rectangular aperture of $8 \times 8 \mathrm{~mm}$.

\section{Analysis of reflectance}

The grade of light polarization at the output the monochromator was different with varying the wavelength, figure 5.2 illustrates spectral dependences of the reflectance, which had been obtained from photodetectors belonging to three different manufacturers. In figures $5.2 a$ and $5.2 b$ that the reflectance of such detectors has a minimum in an area of $1000-1600$ $\mathrm{nm}$, and they both are related to a structure of layers providing maximal responses in the spectral interval of mayor utility of these detectors in near IR optics communication. The first photodiode, see Figure 5a, whose reflectance was minimized, is more efficient that the second one, see figure $5 \mathrm{~b}$.

One can see that this plot presents the other spectrum of reflectance in figure 6, is associated with a photodiode with rectangular aperture. In this case the reflectance has two minima at $1000 \mathrm{~nm}$ and $1600 \mathrm{~nm}$, but the reflectance has a maximum between these minima. This photodiode is older than previous ones, and it was produced by the other manufacturer. One can remark that may be it was produced without good enough control, because the structure of layers on the sensitive surface modifies the reflectance.

The spectrum of reflectance for photodiodes 1 and 4 is presents in figure 7 which belong to the same manufacturer. The reflectance was measured with linearly polarized and nonpolarized lights, and these pair of measurements gives quite similar results. In fact, the difference was equal to approximately $2 \%$. The same results are depicted for the photodiodes 2 and 5, by the second manufacturer. It is important that the results do not depend on the polarization state of the incident light when the angle of incidence is smaller 10 angular degrees.

All spectrums of reflectance for photodiodes 1-6 is presents in the figure 9, with linearly polarized and non polarized lights and is possible to see the different behavior of the photodiodes in the near infrared wavelength.

In fact, in this chapter is studying the behavior of the photodetectors in the near infrared with the linearly polarized and non polarized lights in the case of the polarized lights the angle of incidence is smaller 10 angular degrees and is possible observed it doesn't have changes in the behavior of the reflectance. 


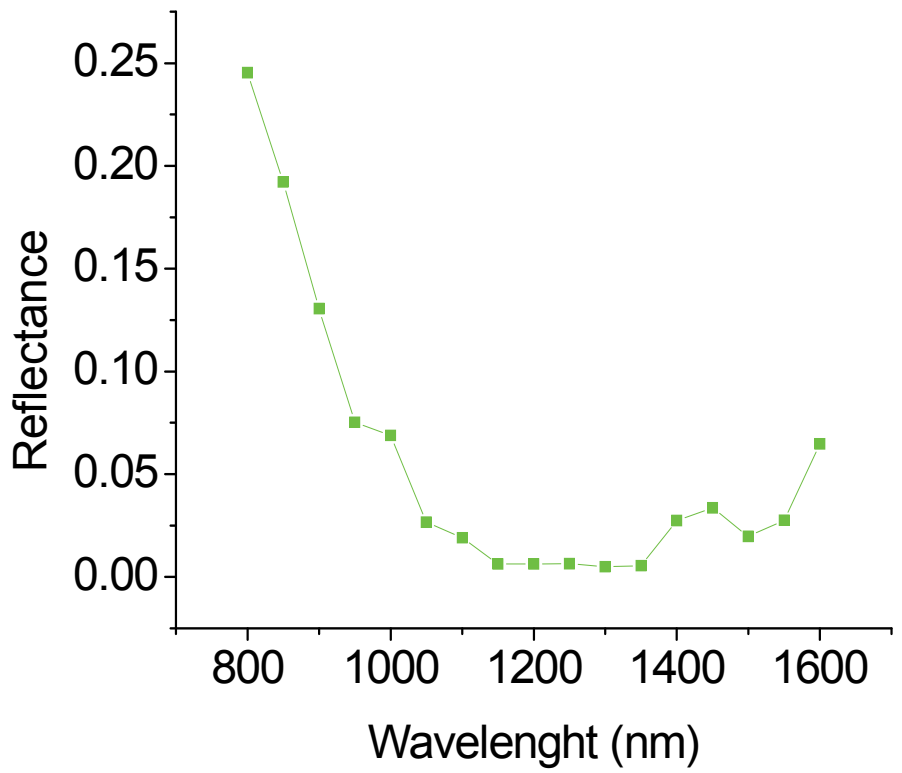

(a)

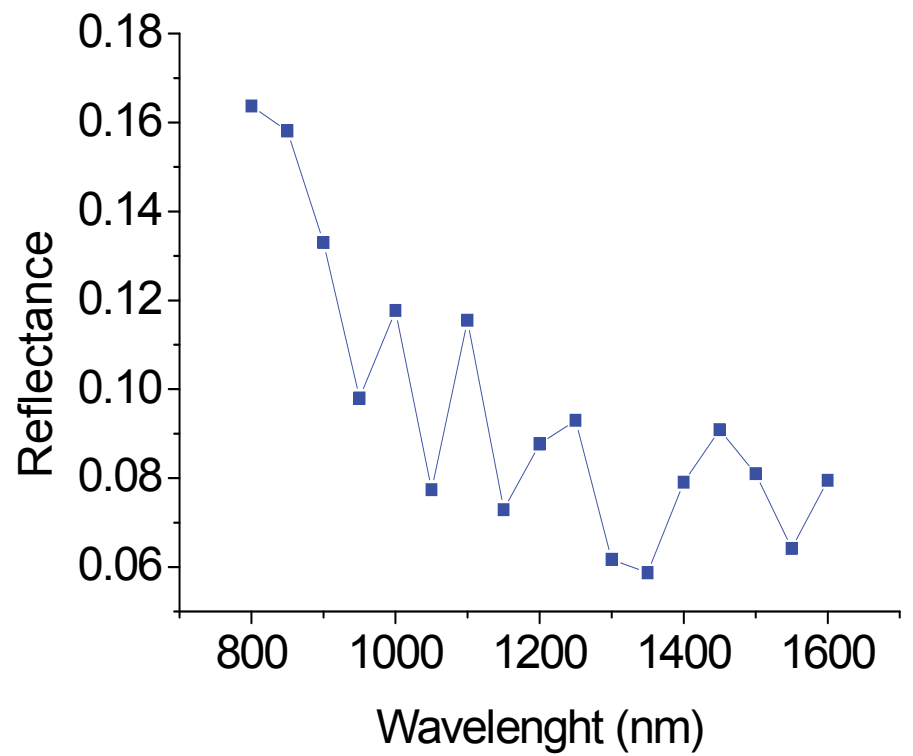

(b)

Fig. 5. Detector with a round aperture with the diameter of $5 \mathrm{~mm}$ 


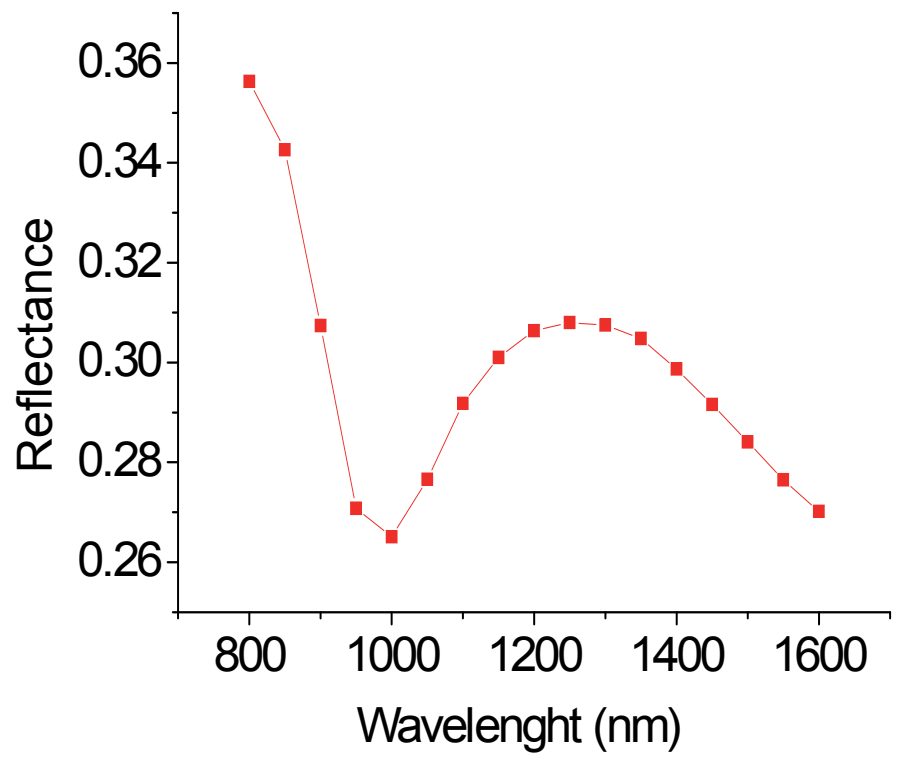

Fig. 6. Detector with a rectangular aperture of $8 \times 8 \mathrm{~mm}$

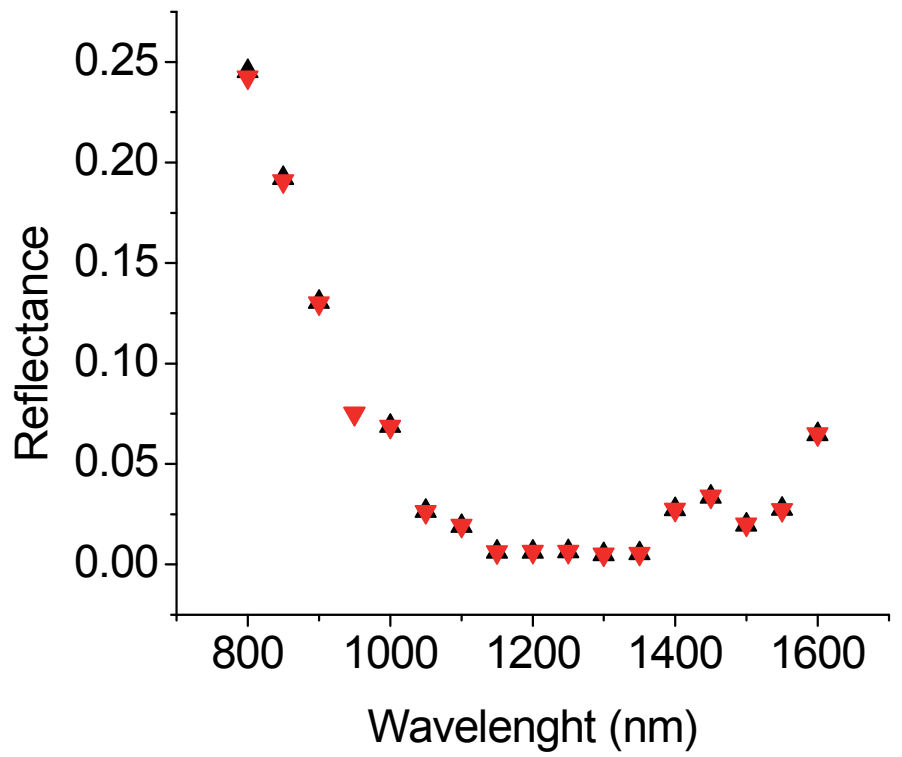

Fig. 7. Spectrum of reflectance for photodiodes 1 and 4 


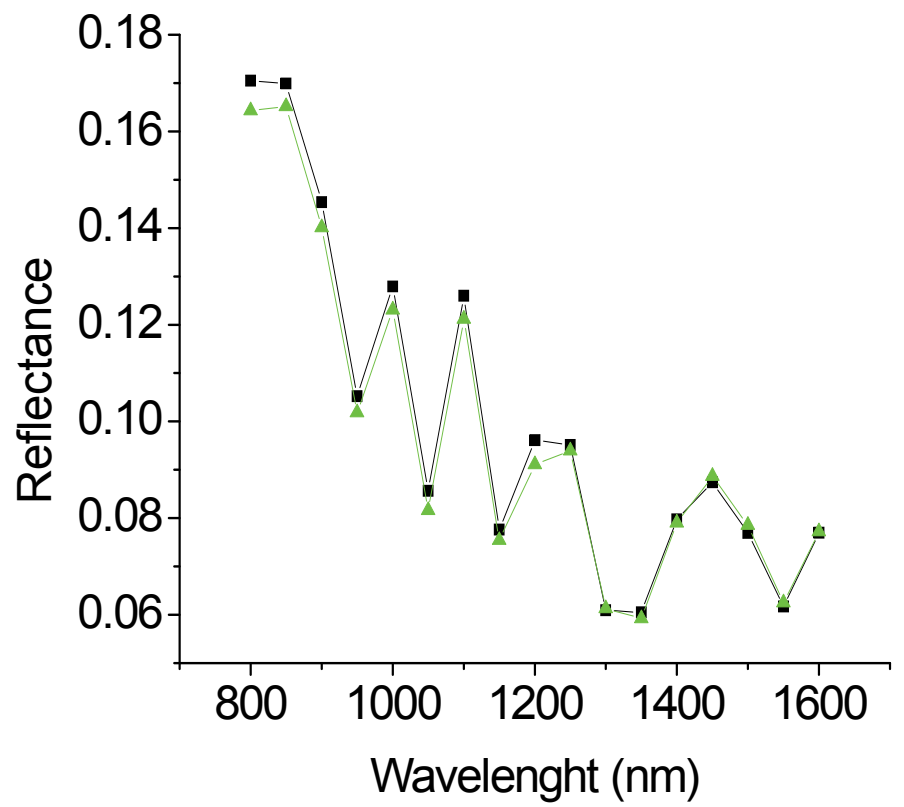

Fig. 8. Spectrum of reflectance of photodiodes 2 and 5.

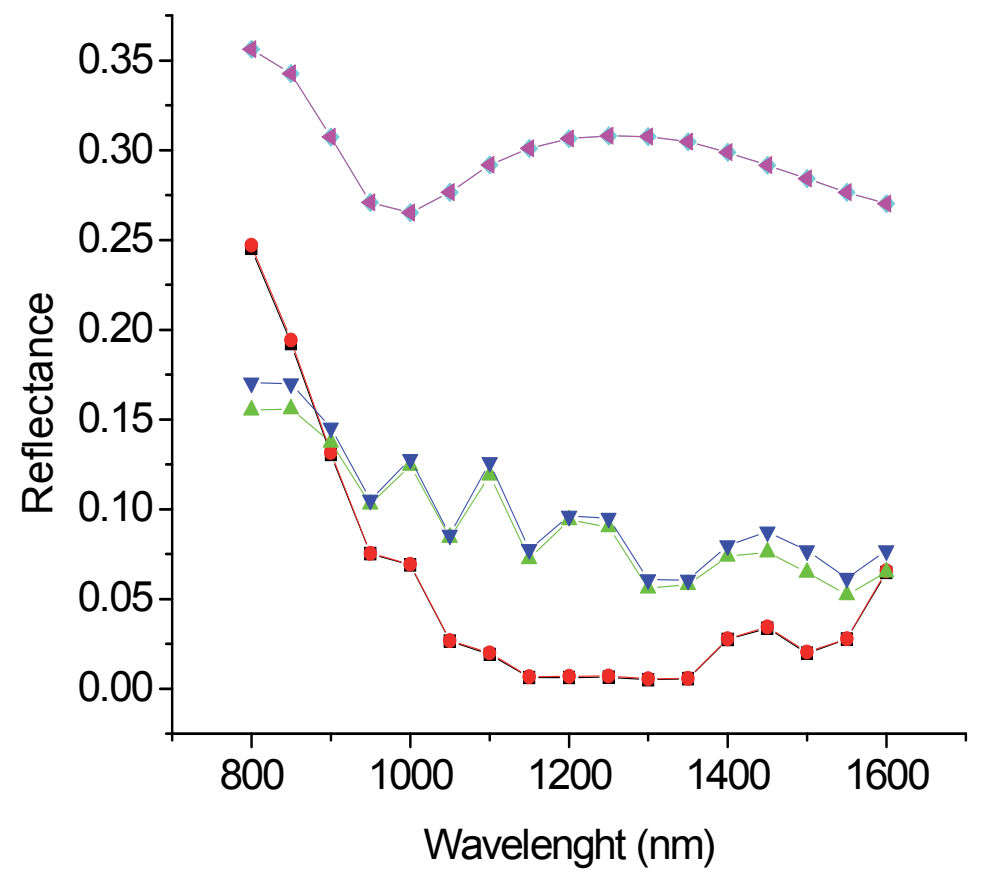

Fig. 9. Spectrum of reflectance of photodiodes 1-6. 


\subsection{External quantum efficiency}

It is obtained from the responsivity values according to the equation:

$$
Q(\lambda) \approx \frac{R(\lambda) h c}{\lambda e}
$$

Where $h, c$ and $e$ are the usual physical constants and $\lambda$ is the wavelength. Values obtained are presented in figure 10 for the same detectors as before. It can be clearly seen that the oldest detector (POL) presents a lower external quantum efficiency than the other and that detector GPD presents a higher external quantum efficiency than detector HAM, which starts to decrease its quantum efficiency at a shorter wavelength.

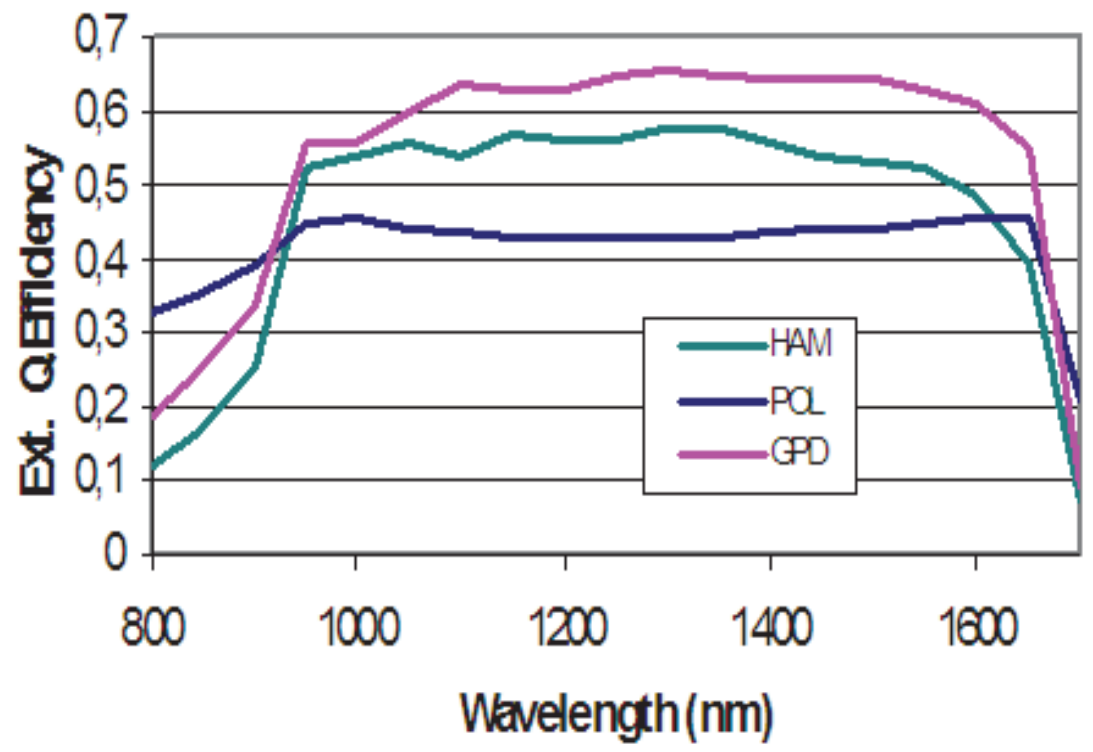

Fig. 10. Spectral external quantum efficiency from responsivity values.

\subsection{Photodiode structure}

Precise structure is not known. The assumed internal structure for photodiodes is shown in figure 11. It is more than likely that detector POL has got a different structure.

Considering for the photodiodes the structure and refraction index values shown (figure 11 and 12), the reflectance can be fitted by using a multilayer model. Results obtained are shown in figure 13, except for photodiode POL that gave a poorer result. The thickness values obtained from the fit are shown in the table. 


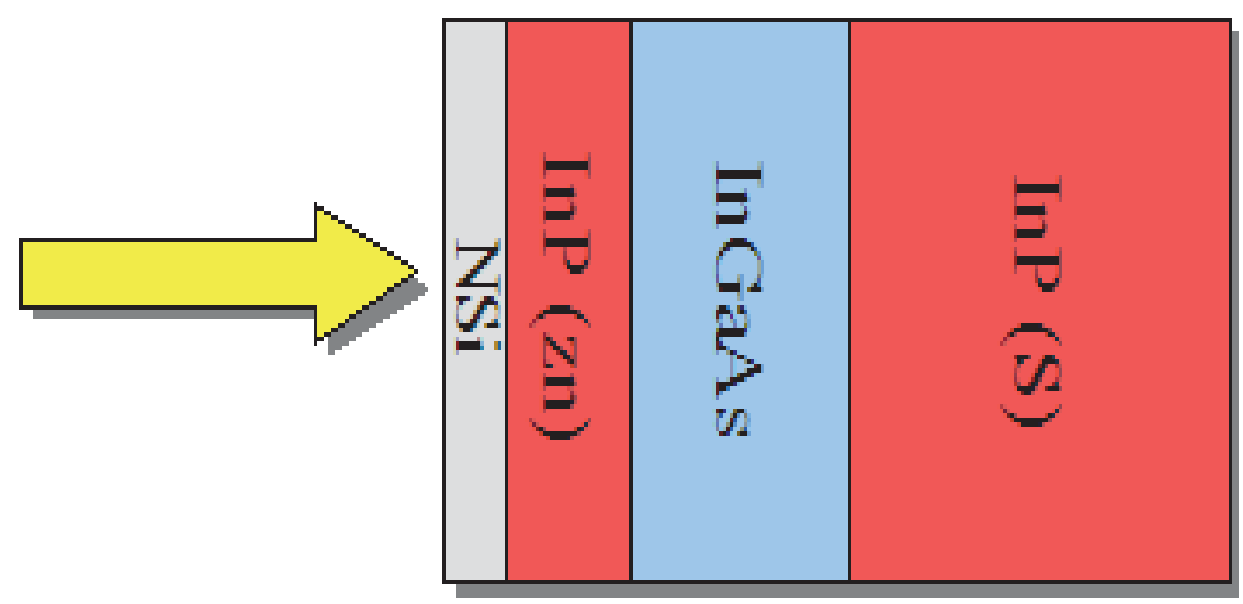

Fig. 11. Possible Structure of InP photodiode.

Spectral Reflectance measured and fitted values for photodiodes HAM and GPD.

\section{Refraction Index}

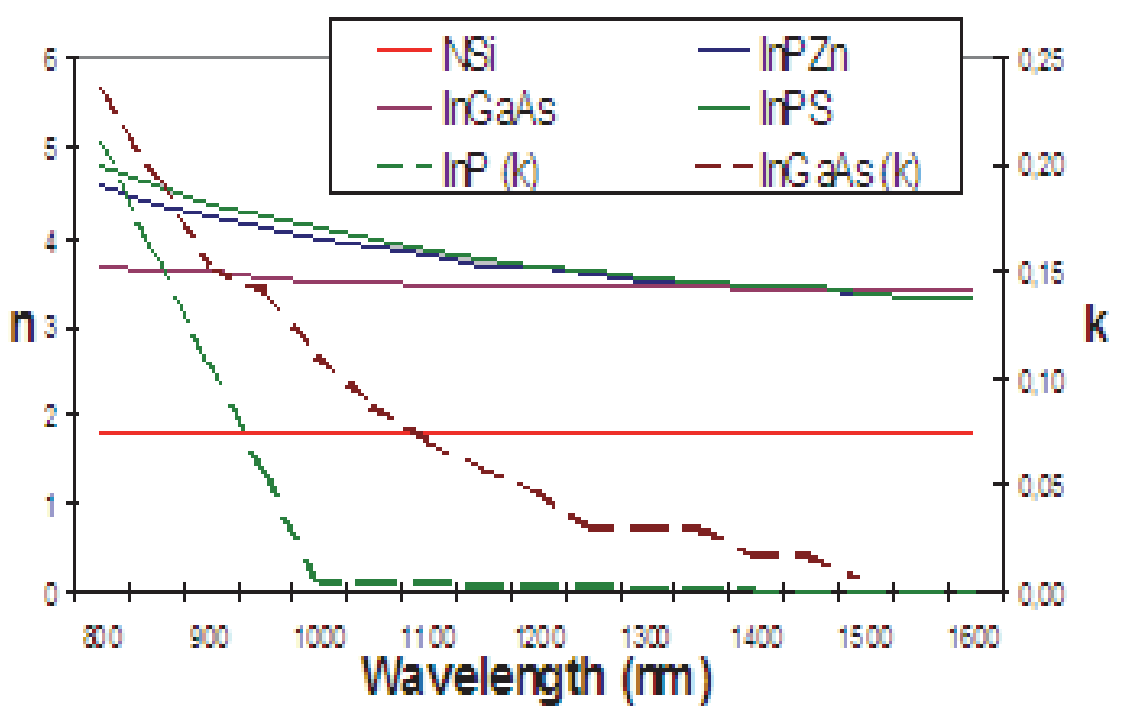

Fig. 12. Materials' Refraction Index 


\begin{tabular}{|c|c|c|c|}
\hline Photodiode & NSi & $\operatorname{InP}(\mathrm{Zn})$ & InGaAs \\
\hline HAM & $162.17 \mathrm{~nm}$ & $1203.35 \mathrm{~nm}$ & $1593.2 \mathrm{~nm}$ \\
\hline GPD & $159.99 \mathrm{~nm}$ & $1200.54 \mathrm{~nm}$ & $1536.7 \mathrm{~nm}$ \\
\hline
\end{tabular}

Table 1 . The thickness values obtained of InP photodiodes.

\subsection{Internal quantum efficiency}

Internal quantum efficiency, $\mathrm{e}(\mathrm{l})$, is calculated as usual: $\mathrm{e}(\mathrm{l})=\mathrm{Q}(\mathrm{l}) /(1-\mathrm{r}(\mathrm{l}))$. Values obtained are shown in figure 13 and 14 for photodiodes HAM and GPD.

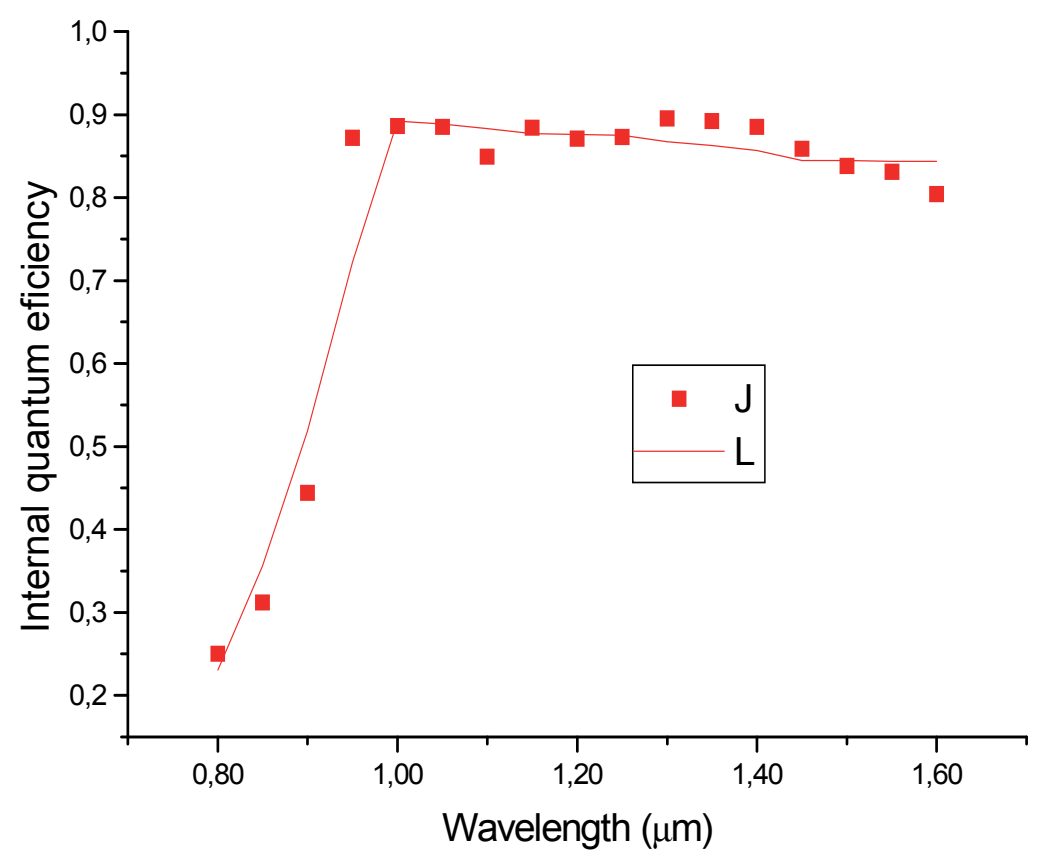

Fig. 13. Internal quantum efficiency of photodiodes HAM experimental values (dots) and fitted values according to the model shown below. 


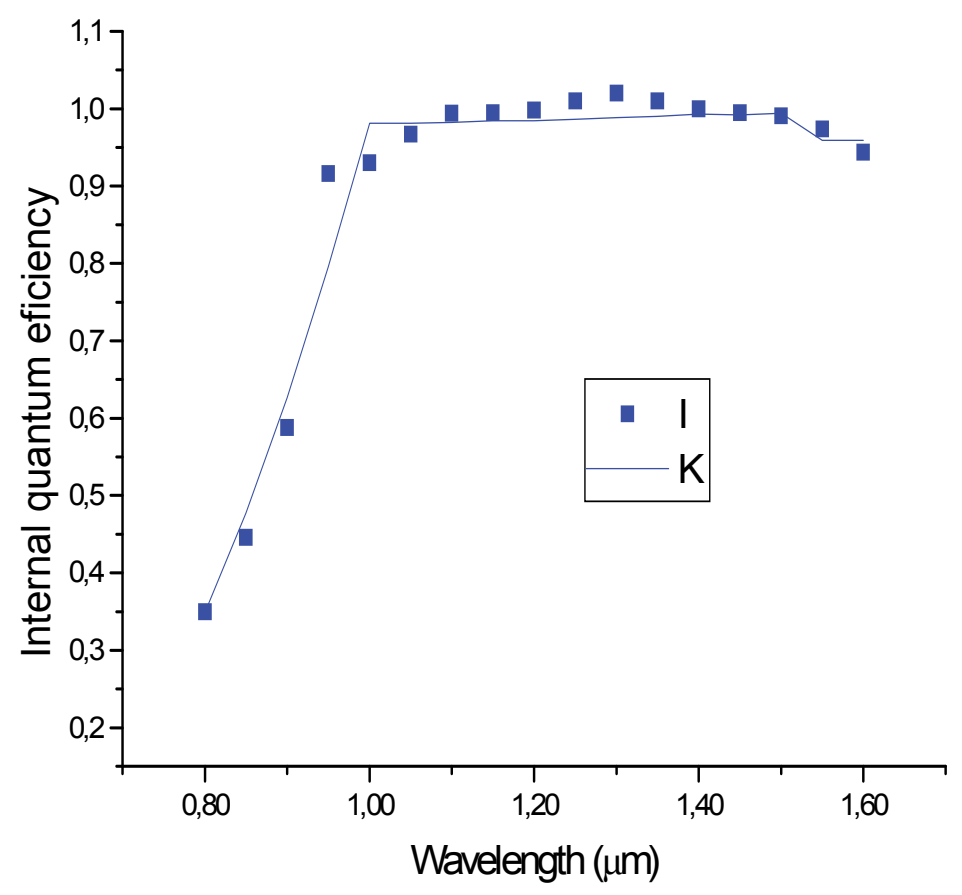

Fig. 14. Internal quantum efficiency of photodiodes GPD experimental values (dots) and fitted values according to the model shown below.

Considering a structure as shown before and a simple model for the collection efficiency of carriers in all regions given by a constant value, Pf,lower than 1 in the first region, 1 in the depletion region and $\mathrm{Pb}$ in the back region, and an "infinite" thickness for the diode, $\mathrm{e}(\mathrm{l}) \mathrm{can}$ be calculated by:

$$
\begin{aligned}
& \varepsilon(\lambda)=P_{f}(1-\exp (-\alpha T))+\exp (-\alpha T)-\exp \left(\alpha T^{\prime}\right)+\exp \left(-\alpha^{\prime} T^{\prime}\right)- \\
& -\exp \left(-\alpha^{\prime} D^{\prime}\right)-\exp \left(-\alpha D^{\prime}\right)+\exp \left(1-P_{b}\right) \exp (-\alpha D)
\end{aligned}
$$

Where $T$ is the thickness at which collection efficiency becomes $1, T^{\prime}$ is the thickness at which InGaAs region starts, $\mathrm{D}^{\prime}$ is the the thickness at which the $\operatorname{InP}(\mathrm{S})$ starts and $\mathrm{D}$ is the thickness at which depletion region ends. By fitting the model to internal quantum efficiency value, the following parameters are obtained for every photodiode.

\begin{tabular}{|c|c|c|c|c|c|c|}
\hline photodiode & Pf & T & $\mathrm{T}^{\prime}$ & $\mathrm{D}^{\prime}$ & $\mathrm{D}$ & $\mathrm{Pb}$ \\
\hline HAM & 0 & 0.44 & 2.19 & 2.19 & 11.96 & 0.844 \\
\hline GPD & 0 & 0.32 & 1.65 & 1.62 & 4351.16 & 0.960 \\
\hline
\end{tabular}

Table 2. Parameters obtained by photodiode 


\section{Conclusions}

The reflectance was measured with linearly polarized and non-polarized lights, and these pair of measurements gives quite similar results. In fact, the difference was equal to approximately $2 \%$. The same results are depicted for the photodiodes 2 and 5 , by the second manufacturer. It is important that the results do not depend on the polarization state of the incident light when the angle of incidence is smaller 10 angular degrees.

In fact in this thesis it are studying the behavior of the photodetectors in the near infrared with the linearly polarized and non polarized lights in the case of the polarized lights the angle of incidence is smaller 10 angular degrees and is possible observed it don't have changes in the behavior of the reflectance.

Reflectance fittinis better for HAM than for GPD. Perhaps the structure model needs some refinement. Internal Quantum Efficiency fitting is not very good at the elbow region. The InP photodiodes' reflectance can be modeled by using a layered optical system having got a first transparent layer and three absorbing ones.

InP photodiodes' internal quantum efficiency can be adequately modeled by a three region structure, where the charge collection efficiency is constant in the two first regions and variable in the last one.

\section{References}

Trommer, R. Steingrüber, R. Löffler, A. Umbach, (2000).Ultrafast, High-Power WaveguidFed Photodetector with Integrated Spot Size Converter, Proc. Of the 12th Int. Conf. On InP and Rel. Mat. (IPRM 2000), Williamsburg, VA, USA, 2000, paper WA2.

A. Beling, H.-G. Bach, G. G. Mekonnen, T. Eckhardt, R. Kunkel, G. Jacumeit, M. Kroh, and J. Berger,(2003) “Fully Packaged InP-based Photodetector for 80/85 Gbit/s RZ Systems," Proceedings 29th European Conference on Optical Communication (ECOC 2003),vol.6, pp. 82-83,September 1-25, 2003, Rimini,Italy.

A. Beling, D. Schmidt, H.-G. Bach, G. G. Mekonnen, R. Ziegler, V. Eisner, M. Stollberg, G. Jacumeit, E. Gottwald, C.-J. Weiske, A. Umbach. (2002). “High power $1550 \mathrm{~nm}$ twin-photodetector modules with $45 \mathrm{GHz}$ bandwidth based on InP", Tech. Digest Optical Fiber Commun. (OFC 2002) , pp.274-275, March 17-22, 2002, Anaheim, CA, USA, paper WN4.

A. H. Gnauck, S. Chandrasekhar, J. Leuthold, and L. Stulz,(2003). "Demonstration of 42.7 Gb/s DPSK receiver with 45 photons/bit sensitivity", IEEE Photon. Technol. Lett., 15 (1), pp. 99-101.

A. Beling, H.-G. Bach, D. Schmidt, G. G.Mekonnen, M. Rohde, L. Molle, H. Ehlers, A. Umbach, "High-Speed Balanced Photodetector Module with 20 dB Broadband Common-Mode Rejection Ratio"(2003). Tech. Digest Optical Fiber Commun. (OFC 2003), vol. 1, pp. 339-340, Atlanta, GA, USA, March 23-28, 2003.

A. Beling, H.-G. Bach, D. Schmidt, G.G. Mekonnen, R. Ludwig, S. Ferber, C. Schubert, C. Boerner, B. Schmauss, J. Berger, C. Schmidt, U. Troppenz and H. G. Weber (2003). "Monolithically integrated balanced photodetector and its application in OTDM 160 Gbit/s DPSK transmission“,Electron. Lett, 39 (16), pp. 1204-1205. 
H.-G. Bach, A. Beling, G. G. Mekonnen, and W. Schlaak,(2002).“Design and fabrication of 60-Gb/s InP-based monolithic photoreceiver OEICs and Modules", IEEE J. Select. Topics Quantum Electron., vol. 8,pp. 1445-1450, Nov./Dec. 2002.

R.Thomas Hawkins II, M. D. Jones,S. H. Pepper, and J. H. Goll, (1991)."Comparison of fast photodetector response measurements by optical heterodyne and pulse response techniques," J. Lightwave Technol, vol. 9,pp. 1289-1294, 1991.

S. Kawanashi, A. Takada, and M. Saruwatari,(1989). "Wide-band frequency response measurement of optical receivers using optical heterodyne detection," J. Lightwave Technol. , vol. 7, No. 1,pp. 92-98, Jan. 1989.

P. Debie and L. Martens,(1995)."Correction technique for on-chip modulation response measurements of optoelectronic devicees," IEEE trans. on Microwave Theory Tech., vol. 43, no. 6, pp. 1264-1269, June 1995.

G. Unterborsch, A. Umbach, D. Trommer, G. G. Mekonnen,(1997)."70GHz long-wavelength photodetector," in Proc.28th Eur. Conf. on Opt. (ECOC '97), pp. 25-28, Sep. 22-25 1997.

G.Wang, T. Tokumitsu,I. Hanawa, Y. Yoneda, K. Sato, and M. Kobayashi,(2003). " A timedelay equivalent-circuit model of ultrafast p-i-n photodiodes," IEEE Trans. on Microwave Tech., vol. 51, No. 4, pp. 1227-1233, April 2003.

A. Siefke, M. Dahlstrom, U. Westergren, X. J. Leijtens, N. van Melick, and M. K. Smit,"Integration of an $8 \times 10 \mathrm{GHz}$ polarization independentWDMreceiver with HBT-preamplifiers," proc. symp. IEEE/LEOS Benelux Chapter,pp. 25-28,Gent, Belgium.

A. Alping," Waveguide pin photodetectors: theoritical analysis and design criteria,(1989)procedins, vol. 136,Pt.J, No. 3, pp. 177-182, June 1989.

O.Wada, H.Hasegawa, K. Taguchi,(2002). "InP Based materials and devices" John Wiley, ISBN: 0-471-18191-9. Unietd States of America.

J. Campos, P. Corredera, A. Pons, A. Corróns J. L. Fontecha.(1998). "Reflectance dependencies of silicon trap detectors". ,IOP Metrología, 35, 455-460.

P Corredera, M L Hernanz, M González-Herráez,J.Campos(2003). “Anomalous non-linear behaviour of InGaAs photodiodes with overfilled illumination" IOP Metrología 40, S181-S184.

P Corredera, M L Hernanz, J Campos, A Corróns, A Pons and J L Fontecha.(2000). "Comparison between absolute thermal radiometers at wavelengths of $1300 \mathrm{~nm}$ and $1550 \mathrm{~nm}$ " IOP Metrología. 37. 237-247.

J.Castrellon, G.Paez, M.Strojnik.(2002) "Radiometric analisis of a fiber optic temperatura sensor" Opt. Eng, 41 (6),1255-1261.

R. Goebel, S. Yilmaz, R.Pello.(1996). "Polarization dependence of trap detectors" IOP Metrología, 33,207-13.

E.F.Zalewski, C.R.Duda, "Silicon photodiode device with $100 \%$ external quantum efficiency" Appl.Opt.,22, 2867-2873.

J.M.Coutin, F.Chandoul, J.Bastie. (2005).“Characterization of new trap detectors as transfer standards" Proceedings of the 9th international conference on new developments and applications in optical radiometry.

A.L.Muñoz Zurita, J.Campos Acosta, A.S.Shcherbakov, A.Pons Aglio.(2006)."Medida de reflectancia y de fotodiodos de InGaAs/InP". Proc. of the VIII National Meeting on Optics, (Optical Society of Spain), p.27-30. 
A.L.Muñoz Zurita, J.Campos Acosta, A.S.Shcherbakov, and A. Pons Aglio.(2007). "Measuring the reflectance and internal quantum efficiency of InGaAs/InP photodiodes in near infrared range". Proc.of the IX International Congress on Advanced Infrared Technology and Applications, (AITA-9), p.1-6.

A.L.Muñoz Zurita, J.Campos Acosta, A.Pons Aglio. A.S.Shcherbakov.(2007). “Medida de la reflectancia de fotodiodos de InGaAs/InP" Óptica Pura y Aplicada, 40(1), 105-109.

A.L.Muñoz Zurita, J.Campos Acosta, A.S.Shcherbakov, and A.Pons Aglio.(2008) “Measuring the reflectance and the internal quantum efficiency of silicon and InGaAs/InP photodiodes in near infrared range". Proc of SPIE Photonics West Conference (January 22-26, 2008, San José, USA).

A.L.Muñoz Zurita, J.Campos Acosta, A.S.Shcherbakov, and A.Pons Aglio. (2008). “External Quantum efficiency of some InGaAs/InP photodiodes", Proc of NewRad2008 congress, MO_P41, (1-3). 


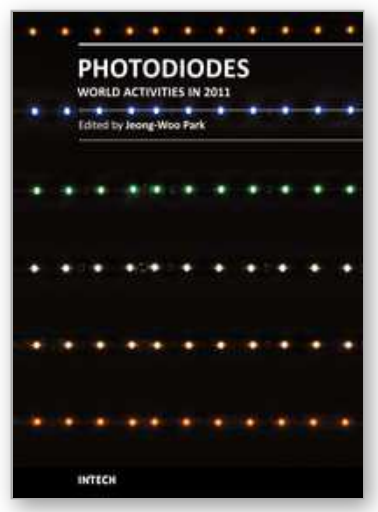

\author{
Photodiodes - World Activities in 2011 \\ Edited by Prof. Jeong Woo Park
}

ISBN 978-953-307-530-3

Hard cover, 400 pages

Publisher InTech

Published online 29, July, 2011

Published in print edition July, 2011

Photodiodes or photodetectors are in one boat with our human race. Efforts of people in related fields are contained in this book. This book would be valuable to those who want to obtain knowledge and inspiration in the related area.

\title{
How to reference
}

In order to correctly reference this scholarly work, feel free to copy and paste the following:

Ana Luz Munoz Zurita, Joaquin Campos Acosta and Alicia Pons Aglio (2011). An Absolute Radiometer Based on InP Photodiodes, Photodiodes - World Activities in 2011, Prof. Jeong Woo Park (Ed.), ISBN: 978-953-307530-3, InTech, Available from: http://www.intechopen.com/books/photodiodes-world-activities-in-2011/anabsolute-radiometer-based-on-inp-photodiodes

\section{INTECH}

open science | open minds

\section{InTech Europe}

University Campus STeP Ri

Slavka Krautzeka 83/A

51000 Rijeka, Croatia

Phone: +385 (51) 770447

Fax: +385 (51) 686166

www.intechopen.com

\section{InTech China}

Unit 405, Office Block, Hotel Equatorial Shanghai

No.65, Yan An Road (West), Shanghai, 200040, China 中国上海市延安西路65号上海国际贵都大饭店办公楼 405 单元

Phone: +86-21-62489820

Fax: +86-21-62489821 
(C) 2011 The Author(s). Licensee IntechOpen. This chapter is distributed under the terms of the Creative Commons Attribution-NonCommercialShareAlike-3.0 License, which permits use, distribution and reproduction for non-commercial purposes, provided the original is properly cited and derivative works building on this content are distributed under the same license. 